\title{
Fungi pathogenic to humans: molecular bases of virulence of Candida albicans, Cryptococcus neoformans and Aspergillus fumigatus ${ }^{\star}$
}

\author{
Justyna Karkowska-Kuleta $\bowtie$, Maria Rapala-Kozik and Andrzej Kozik \\ Department of Analytical Biochemistry, Faculty of Biochemistry, Biophysics, and Biotechnology, Jagiellonian \\ University, Kraków, Poland
}

Received: 07 April, 2009; revised: 06 June, 2009; accepted: 17 June, 2009 available on-line: 18 June, 2009

\begin{abstract}
The frequency of severe systemic fungal diseases has increased in the last few decades. The clinical use of antibacterial drugs, immunosuppressive agents after organ transplantation, cancer chemotherapy, and advances in surgery are associated with increasing risk of fungal infections. Opportunistic pathogens from the genera Candida and Aspergillus as well as pathogenic fungi from the genus Cryptococcus can invade human organism and may lead to mucosal and skin infections or to deep-seated mycoses of almost all inner organs, especially in immunocompromised patients. Nowadays, there are some effective antifungal agents, but, unfortunately, some of the pathogenic species show increasing resistance. The identification of fungal virulence factors and recognition of mechanisms of pathogenesis may lead to development of new efficient antifungal therapies. This review is focused on major virulence factors of the most common fungal pathogens of humans: Candida albicans, Aspergillus fumigatus and Cryptococcus neoformans. The adherence to host cells and tissues, secretion of hydrolytic enzymes, phenotypic switching and morphological dimorphism contribute to $C$. albicans virulence. The ability to grow at $37^{\circ} \mathrm{C}$, capsule synthesis and melanin formation are important virulence factors of $C$. neoformans. The putative virulence factors of $A$. fumigatus include production of pigments, adhesion molecules present on the cell surface and secretion of hydrolytic enzymes and toxins.
\end{abstract}

Keywords: pathogenic fungi, virulence factors, aspergillosis, candidiasis, cryptococcosis

\section{INTRODUCTION}

In the last decades the problem of severe nosocomial fungal diseases has become more serious, especially in patients with severe immunological impairment. The development of medicine, surgery and transplantology in the last thirty years has caused a dramatic increase in the number of immunocompromised individuals who are more susceptible to fungal infections. Patients with immunological impairment, HIV infection, leukopenia (haematological malignancy patients), after surgery, organ trans- plantation or cancer therapy are at risk of developing mycoses. Widespread use of broad-spectrum antimicrobial agents, immunosuppressive agents and corticosteroid therapy are also risk factors. The prophylactic use of antifungal therapies is one of the reasons of frequent resistance to antifungal drugs (Perfect \& Casadevall, 2006; d'Enfert \& Hube, 2007). Among all the fungi only few species are pathogenic to humans. The most frequently diagnosed fungal infections are caused by pathogens from the genera Candida, Cryptococcus and Aspergillus (Richardson, 2005). These fungi are ubiquitous and can be

$\square$ Corresponding author: Justyna Karkowska-Kuleta, Department of Analytical Biochemistry, Faculty of Biochemistry, Biophysics, and Biotechnology, Jagiellonian University, Gronostajowa 7, 30-387 Kraków, Poland; tel.: (48) 12 664 6544; fax: (48) 12664 6902; e-mail: justyna.karkowska@uj.edu.pl

^Presented at the XXXVI Winter School "Molecule interactions in health and disease" organized by the Faculty of Biochemistry, Biophysics and Biotechnology, Jagiellonian University, 21-26 February, 2009, Zakopane, Poland.

Abbreviations: ALS, agglutinin-like sequence; GlcNAc, N-acetyl-D-glucosamine; GPI, glycosylphosphatidylinositol; GXM, glucuronoxylomannan; HBMEC, the human brain microvascular endothelial cells; MAP, mitogen-activated protein; PMN, polymorphonuclear neutrophils; RGD, Arg-Gly-Asp adhesion sequence; SAPs, secreted aspartyl proteinases; SIR2, Silent mating type Information Regulation-2. 
acquired from host surroundings (Cryptococcus neoformans, Aspergillus fumigatus) or are components of normal endogenous flora (Candida albicans) (Perfect \& Casadevall, 2006). The mortality among infected patients is high, even after intensive antifungal treatment, because of patient's immunodeficiency, late diagnosis or fungal drug resistance. Fungi are able to cause a disease and to overwhelm the host defense systems because of possessing several genes and proteins associated with their pathogenicity, called virulence factors (Tomee \& Kauffman, 2000). Many of the putative fungal virulence factors have developed naturally during organism evolution and originally acted as a defense against unfavorable environmental conditions, and then, in this way, many of them became important as virulence factors facilitating infection.

\section{CANDIDA ALBICANS}

Currently more than two hundred ascomycetous yeasts are included into the genus Candida, but only a few species are opportunistic pathogens of humans. Nowadays, Candida albicans is thought to be the major fungal pathogen of humans. Severe Candida infections are a serious problem, especially in individuals whose immune defense mechanisms have been weakened (Odds et al., 2006). C. albicans can colonize skin and mucosal surfaces of healthy people and thus occurs commensally in the gastrointestinal tract, oral cavity and vagina, often causing superficial infections (Mavor et al., 2005). Moreover, C. albicans can enter the bloodstream by direct penetration from the epithelium after tissue damage, or by dissemination from biofilms formed on medical devices introduced into the patient's organism, e.g. catheters, dental implants, endoprostheses, artificial joints or central nervous system shunts (Chandra et al., 2001; Mavor et al., 2005). Then yeast cells disseminate with the blood flow and infect almost all inner organs, including lungs, kidney, heart, liver, spleen and brain, causing fungaemia and life-threatening septicaemia. Candidiasis may occur as a result of disturbed balance between host immunity and this opportunistic pathogen. This disorder is not only due to the immunological dysfunction of the host, but also to the fungal ability to adapt to new niches, dependent on the expression of infection-associated genes (Brown et al., 2007a). These genes and their products contribute to fungal pathogenicity and are described as virulence factors. C. albicans virulence factors include, among others, production of different hydrolytic enzymes and adhesins (Chaffin et al., 1998). There are also other characteristic properties that influence fungal virulence, for example, the ability to form biofilms on various surfaces, to change morphological form and to switch between various phenotypes (Chaffin et al., 1998).

\section{Phenotypic switching}

Phenotypic switching is a very important part of fungal adaptability to the changing of environment during invasion of the human organism. The ability to infect many tissues is crucial to a successful attack and dissemination within the host. Occasionally some subpopulations of $C$. albicans cells can change their morphology, cell surface properties, colony appearance, biochemical properties and metabolism to become more virulent and more effective during infection (Odds et al., 2006). Colonies change their appearance and assume different shapes, including smooth, rough, fuzzy, wrinkled, fringed or stippled phenotype with a high frequency, approximately one changed colony per $10-10^{4}$ colonies (Slutsky et al., 1985). The molecular basis of this process is still unclear. Probably chromosomal rearrangements and a SIR2-like regulation take part in this process (Calderone \& Fonzi, 2001). The most popular and well-known example of switched colonies is the white - opaque switching, when a white, oval and smooth colony changes into a grey, rough colony (Slutsky et al., 1987). The opaque cells produce aspartyl proteinases 1 and 3 and are less virulent, whereas white cells secrete aspartyl proteinase 2 and are more virulent during systemic infection (Yang, 2003). Phenotypic switching is most likely a signal of large-scale processes involving changes of many molecular and biochemical properties of the pathogen, which are helpful for fungi to survive within the host organism.

\section{Morphological dimorphism}

The ability to switch between unicellular yeast cells and filamentous forms called hyphae and pseudohyphae is known as morphological dimorphism. The transition between these different morphological forms in response to diverse stimuli seems to be very important for fungal pathogenicity (Lo et al., 1997; Chaffin et al., 1998). The morphology can change under a variety of environmental conditions, including response to physiological temperature of $37^{\circ} \mathrm{C}, \mathrm{pH}$ equal to or higher than 7.0, $\mathrm{CO}_{2}$ concentration of $5.5 \%$ or the presence of serum or carbon sources which stimulate hyphal growth (Eckert et al., 2007). The production of unicellular forms is stimulated by lower temperatures and more acidic $\mathrm{pH}$, absence of serum and high concentrations of glucose (Whiteway \& Bachewich, 2006). Yeast cells are thought to be responsible for dissemination in the environment and finding new hosts, while hyphae are required for tissue damage and invasion. 
Both forms are present in biofilms formed on artificial substrates (Chandra et al., 2001). Yeast cells have different properties than the mycelial forms: the ultrastructure, biological attributes and composition of the cell wall differ between these forms. Probably both forms, yeast cells and hyphae, are necessary for full virulence, because mutants lacking genes responsible for the production of one or the other are less virulent (Yang, 2003). Despite the fact that the production of filamentous form is well known among the pathogenic fungi, the molecular basis of C. albicans morphological dimorphism is still poorly understood. Many genes associated with the formation of different cell shape code for transcription factors and may be also responsible for expression of unknown genes, encoding other virulence factors. Recent studies show that the transcription factor Cph1p, whose phosphorylation is regulated through a mitogen-activated protein (MAP) kinase pathway involving products of genes CST20, HST7 and CEK1, and the basic helix-loop-helix transcription factor Efg1p are required to form hyphae during infection (Lo et al., 1997). In the Efg1p pathway, the following proteins are involved: homologs of Ras, adenyl cyclase and protein kinase A (TPK2) (Brown \& Gow, 1999; Lengeler et al., 2000). Other transcription factors, Tup1p and $\mathrm{Rbp} 1 \mathrm{p}$, are negative regulators of filamentation, because mutants lacking these genes exhibit constitutive filamentation (Calderone \& Fonzi, 2001; Yang, 2003). Other putative factors may contribute to morphogenesis, but their role and exact function are so far unknown. It is only obvious that co-operation of all the signaling pathways involved in the morphological transition is very important for fungi to infect and survive in the human host.

\section{Adhesion and adhesion molecules}

The adherence to the host cells and tissues, as well as the binding of a set of diverse host proteins is essential for $C$. albicans to begin the invasion, followed by dissemination within the human organism. This step is crucial for fungal survival. On the cell wall surface $C$. albicans presents receptors which are responsible for adhesion to epithelial and endothelial cells, serum proteins and extracellular matrix proteins (Chaffin et al., 1998). Adhesion to different artificial substrates and formation of biofilm on medical devices is currently a serious problem in medicine, because of the frequent resistance to antifungal agents and increased pathogenicity among the subpopulation of cells forming the biofilm. It has been estimated that in the last few decades microbial infections of humans are strictly correlated with biofilm formation in $65 \%$ of cases (Ramage et al., 2006). C. albicans cells originally present either on the skin, mucosal surfaces or in blood can colonize the sur- face of medical devices. All known morphological forms, yeast cells, pseudohyphae and hyphae, form a biofilm and they have different properties than those of planktonic or suspended cells (Al-Fattani \& Douglas, 2006). The secretion of aspartyl proteinases (SAPs) is higher during biofilm formation (Mendes et al., 2007). C. albicans cells forming a biofilm are always associated with a matrix composed of polysaccharides containing mannose and glucose residues (Chandra et al., 2001). The biofilm matrix production plays a very important role in drug resistance of C. albicans biofilms, but development of resistance is rather multifactorial (Al-Fattani \& Douglas, 2006). During biofilm formation, C. albicans cells express several genes that influence pathogenicity. Products of these genes take part in adhesion (e.g. family of Als proteins), in carbohydrate synthesis, drug resistance (e.g. efflux pumps) and in quorum sensing (Chandra et al., 2001).

The ability of Candida to invade different environments in the host organism is a result of great flexibility and adaptability of fungi. This phenomenon is in part due to the presence of different adhesins connected with cell surface, which facilitate the first stage of infection. These adhesins include Als proteins family, Hwp1p, Eap1p, Csh1p, and other less known cell surface receptors. The $C$. albicans cell wall is constructed from $\beta$-glucans (branched polymers of glucose residues containing $\beta-1,3$ and $\beta-1,6$ linkages), chitin (unbranched polymers of $\mathrm{N}$-acetylD-glucosamine (GlcNAc) containing $\beta-1,4$ bonds), mannoproteins, small amounts of other proteins and lipids (Chaffin et al., 1998). All known receptors are tightly connected with the fungal cell wall. The family of Als proteins ("agglutinin-like sequence") is one of the well-known examples of Candida adhesins. This family includes at least eight ALS genes that encode proteins with similar structure, which are connected to the cell surface (Fig. 1). Despite the fact that members of this family have different functions and different sizes, their structures exhibit some common properties. In 2004, Sheppard and coworkers described the structure of the N-terminal fragment of Als proteins. This part contains multiple antiparallel $\beta$-sheet domains containing minor $\alpha$ helical and $\beta$-turn components, which indicates that products of $A L S$ genes are members of immunoglobulin family (Sheppard et al., 2004). Als1p, Als3p and Als5 $p$ are believed to be responsible for adherence to collagen, fibronectin, laminin, endothelial and epithelial cells, Als6p binds to collagen and Als9p to laminin. Als4p probably mediates adherence to endothelium, and Als5p is additionally responsible for cell-to-cell aggregation. The role of Als7p is still unknown (Filler et al., 2006). A receptor with a molecular mass of approx. $34 \mathrm{kDa}$, which is present only on the surface of hyphal cells, is called Hwp1p 


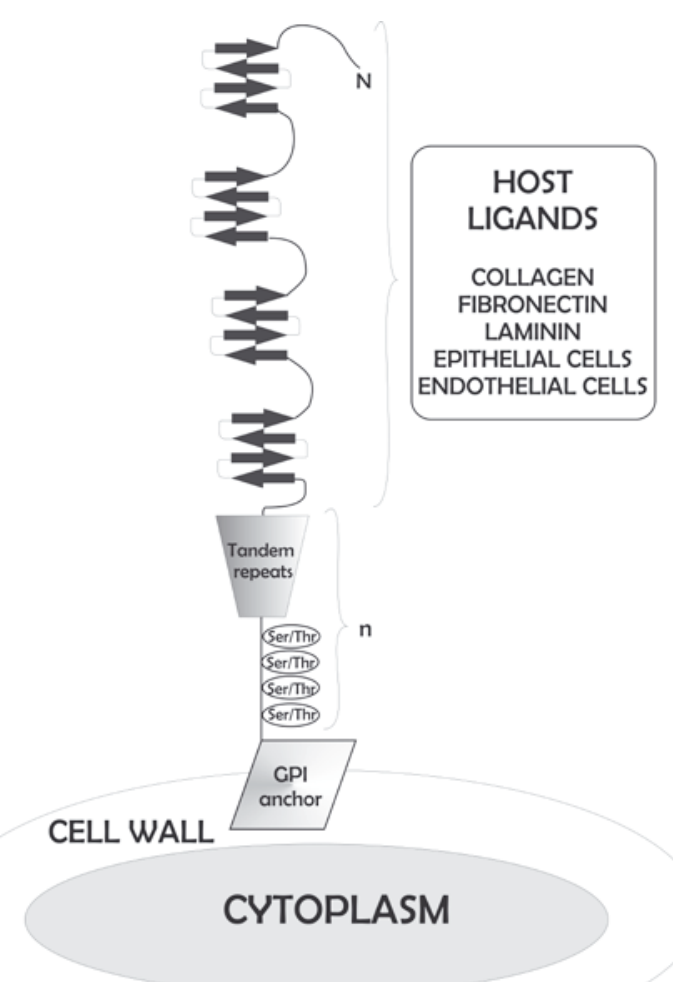

Figure 1. Scheme of Als adhesin structure.

The N-terminal fragment contains a putative signal peptide and a ligand-binding domain, central region is rich in tandem repeats with many serine and threonine residues, as well as consensus sites for glycosylation. The C-terminal part contains a glycosylphosphatidylinositol (GPI) anchorage site. After Filler et al. (2006), modified.

(Chaffin et al., 1998). The structure of Hwp1p is similar to that characteristic for Als proteins, but unlike those, Hwp1p possess in its N-terminal part a sequence similar to those of small proline-rich proteins of human cells which are substrates for transglutaminases present on the surface of epithelial cells. Therefore, Hwp1p facilitates adhesion to epithelium (Staab et al., 1999). Another receptor, Eap1p ("enhanced adhesion to polystyrene 1"), shows homology and structural similarity to Hwp1p, but the host ligands for this protein are still unknown (Filler et al., 2006). There are also other receptors of different structure, for example Int1p containing an integrin-like domain and an RGD-binding domain, which bind to different extracellular matrix proteins including fibronectin, entactin, vitronectin, laminin and collagen, and take part in adhesion to platelets and endothelial cells (Chaffin et al., 1998; Calderone \& Fonzi, 2001; Ruiz-Herrera et al., 2006). Although the role of alcohol dehydrogenase, Adh1p, in binding of $C$. albicans cells to fibronectin is still unclear this protein is also thought to have adhesive properties (Filler et al., 2006). Host serum proteins, includ- ing fibrinogen (Casanova et al., 1992), plasminogen (Crowe et al., 2003), kininogen (Rapala-Kozik et al., 2008) and others can also be bound by C. albicans cells through different receptors, from which some are known and partially characterized. A tighter adherence to epithelial and endothelial cells, as well as to extracellular matrix proteins is achieved thanks to increased cell surface hydrophobicity. Singleton et al. (2001) have described a novel 38-kDa receptor, Csh1p, which enhances hydrophobicity of C. albicans cells, also facilitating specific receptor-ligand interactions. Adhesion to host cells is also dependent on interactions between mannoproteins with lectin-like properties and fucosyl or glucosaminyl glycosides on epithelial cells' surface (Ruiz-Herrera et al., 2006). Although many fungal adhesins have been identified, so far little is known about the host receptors involved in adhesion. At present it is obvious that Toll-like receptors 2 and 4 and other receptors present on the surface of human immune cells, such as monocytes, macrophages and dendritic cells, are involved in these interactions. Probably some proteins present on the surface of epithelial or endothelial cells, for example N-cadherin, are important for binding of different morphological forms of C. albicans cells, followed by their endocytosis (Phan et al., 2005; Filler, 2006). Due to the ability to bind and invade human endothelial cells $C$. albicans can traverse the human blood-brain barrier and cause life-threatening meningitis (Jong et al., 2001).

\section{Secreted hydrolytic enzymes}

Production and secretion of hydrolytic enzymes, such as proteases, lipases and phospholipases are very important virulence factors. These enzymes play a role in nutrition but also in tissue damage, dissemination within the human organism, iron acquisition and overcoming the host immune system, and strongly contribute to fungal pathogenicity. Many types of secreted hydrolytic enzymes are currently known for $C$. albicans. The activity of phospholipases is very high during tissue invasion, because these enzymes are responsible for hydrolysis of one or more ester linkages of glycerophospholipids, of which the cell membrane is built. C. albicans cells isolated from blood produce higher extracellular phospholipase activities than commensal strains (Ibrahim et al., 1995). There are four types of secreted phospholipases: A, B, C and D (Calderone \& Fonzi, 2001; Yang, 2003), specific towards individual ester bonds in glycerophospholipids (Ghannoum, 2000). Very important for fungal virulence is the activity of phospholipase B (PLB), which has both hydrolase and lysophospholipase-transacylase activities (Ghannoum, 2000; Yang, 2003). Thus, PLB can release fatty acids from a phospholipid and the 
remaining fatty acid from a lysophospholipid, and then transfer a free fatty acid to a lysophospholipid and produce phospholipids (Theiss et al., 2006). Apart from phospholipases, C. albicans can produce at least nine lipases which can hydrolyze ester bonds of mono-, di-, and triacylglycerols (Schaller et al., 2005). A well-known group of $C$. albicans secreted hydrolytic enzymes are SAPs (secreted aspartyl proteinases). The family of $S A P$ genes includes at least ten different genes SAP1-SAP10 which encode enzymes with similar functions and character, but different molecular properties, such as molecular mass, isoelectric point and $\mathrm{pH}$ for optimal activity (Naglik et al., 2003). The expression of the SAP genes is regulated at the transcriptional level, and the nascent preproprotein is processed by a signal peptidase in the endoplasmatic reticulum and by a Kex2-like proteinase at the carboxyl-terminal side in the Golgi apparatus (Newport \& Agabian, 1997; Yang, 2003). The mature enzymes have molecular masses in the range of $35-48 \mathrm{kDa}$ and two highly conserved regions with reactive aspartic residues, just as other pepsinelike proteinases (Schaller et al., 2005). Probably SAPs 1-3 are secreted only by yeast cells and SAPs $4-6$ by hyphal forms (Naglik et al., 2004; White \& Agabian, 1995), whereas both forms produce SAPs 9 and 10, which are connected with fungal cell walls because of possessing a GPI anchorage site (Hube \& Naglik, 2001; Albrecht et al., 2006). The synthesis and function of SAPs 7 and 8 are still under investigation (Yang, 2003). Many host proteins are hydrolyzed by secreted aspartyl proteinases, including collagen, laminin, fibronectin, mucin, salivary lactoferrin, $\alpha_{2}$-macroglobulin, almost all immunoglobulins, the proinflammatory cytokine interleukin- $1 \beta$, lactoperoxidase, cathepsin D, complement, cystatine A, and precursors of several blood coagulation factors (Fig. 2) (Naglik et al., 2004; Schaller et al., 2005). The spectrum of optimal $\mathrm{pH}$ for SAPs activity is from 2.0 to 7.0, therefore these enzymes may contribute to fungal pathogenesis and developing infections in different sites in the human organism (Naglik et al., 2004). Except aspartyl proteinases, C. albicans also secrets other proteases: a $60-\mathrm{kDa}$ metallopeptidase and a 50-kDa serine peptidase. The serine peptidase is active in a broad range of $\mathrm{pH}(5.0-7.2)$ and hydrolyzes many host substrates including extracellular matrix proteins and serum proteins (dos Santos et al., 2006).

\section{Other virulence factors}

The ability of pathogenic microorganisms to acquire iron from the environment during infection is another very important virulence factor. The ability to overcome host systems connected with iron transport and accumulation is crucial for the patho-

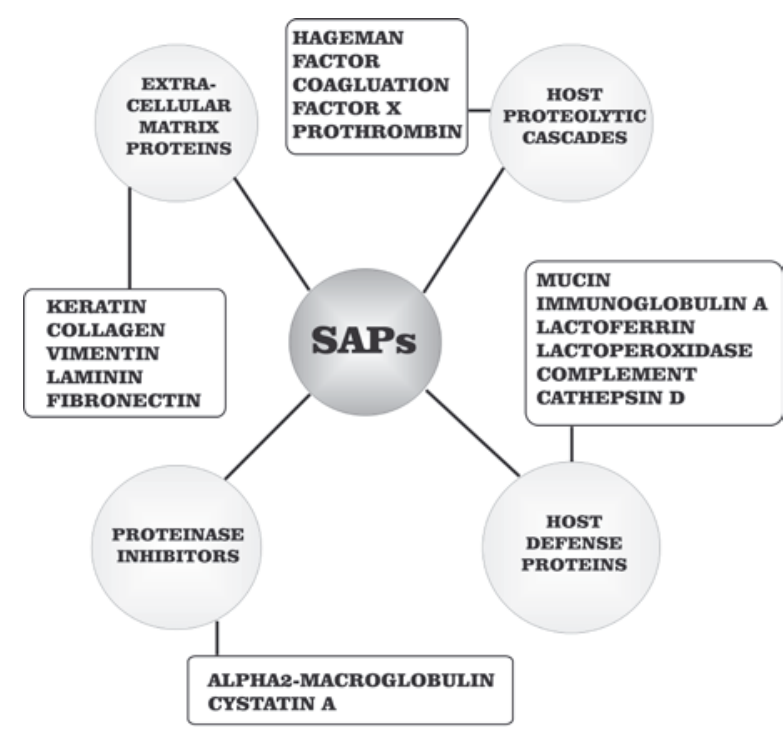

Figure 2. Substrates hydrolyzed by secreted aspartyl proteinases (SAPs) during infection.

Based on data of Naglik et al. (2003) and Schaller et al. (2005).

gen to survive during invasion of the bloodstream. In C. albicans members of Rbt5 family are needed for utilization of hemoglobin and hemin for iron acquisition by the pathogen. Without these proteins the $C$. albicans iron metabolism is severely impaired (Weissmen \& Kornitzer, 2004). During infection Candida cells are exposed to reactive oxygen species produced by immune cells, hence the organism expresses several virulence factors which help to overcome this host defense mechanism, including catalase, superoxide dismutase and heat shock proteins (Brown et al., 2007a). Expression of many virulence factors often depends on environmental conditions, therefore fungi must possess a sensor for environmental changes. Probably calcineurin plays the role of such a sensor. Calcineurin is a highly conserved protein involved in fungal stress responses, composed of two subunits, the A subunit with catalytic activity and the B subunit with a regulatory function (Blankenship et al., 2003). The catalytic subunit is encoded by the CMP1 gene (Bader et al., 2003). As a result of calcium influx calmodulin binds to calcineurin A subunit, inhibits the action of the autoinhibitory C-terminal domain of the A subunit, and leads to the formation of the active calcineurin complex, which has a protein phosphatase activity. Afterwards calcineurin may influence the expression of several virulence factors of $C$. albicans. In the case of C. albicans it was shown by Blankenship et al. (2003) that calcineurin is dispensable for growth at $37^{\circ} \mathrm{C}$, germ tube formation, and adherence to the host cells, but is essential for survival in the human serum, so fungal pathogenicity strongly correlates with its activity. 


\section{CRYPTOCOCCUS NEOFORMANS}

Cryptococcus neoformans is a saprophytic, basidiomycetous, dimorphic organism found worldwide, because its natural habitats are pigeon droppings and contaminated soil. Small-sized basidiospores (1.8 to $3.0 \mu \mathrm{m}$ ) (Buchanan \& Murphy, 1998; Lin \& Heitman, 2005) can turn into yeast cells, the form preferred at $37^{\circ} \mathrm{C}$, or can form dikaryotic hyphae which are favored at $24^{\circ} \mathrm{C}$ (Whiteway \& Bachewich, 2006). The haploid yeast cells are the asexual form of $C$. neoformans found in tissues during fungal infection as well as in standard laboratory media. In contrast, the diploid sexual form, hyphae, which is unstable and transient, is found during culture of MAT $\alpha$ and MATa strains on minimally nutritious media such as V-8 juice agar (Sia et al., 2000; Perfect, 2006). The $\alpha$ strains, which are thought to be more virulent than a strains, can undergo a true dimorphic transition from haploid yeast cells to haploid hyphal cells producing viable basidiospores (Wickes et al., 1996). Basidiospores or yeast cells may be inhaled by humans, then through the respiratory tract the pathogen can disseminate within the organism causing pulmonary infections, and subsequently, due to the C. neoformans predilection for the central nervous system, the lifethreatening meningoencephalitis both in immunocompromised and immunocompetent patients. If the infection of the central nervous system is not cured, it is fatal in 100\% of cases (Buchanan \& Murphy, 1998), and even after treatment the mortality rate is between $10 \%$ and $25 \%$ (Perfect \& Casadevall, 2002). Since the human immune system is in almost all cases able to overcome the intruder, infections can often pass without symptoms, but sometimes even immunocompetent patients can contract a severe disease. In patients after surgery, organ transplantation, with HIV infection or other malignancies, infections are more serious and lead to high mortality (Mitchell \& Perfect, 1995; Perfect \& Casadevall, 2002). This fungal pathogen is less known than Candida species, but nowadays the morbidity and mortality caused by cryptococcosis is a significant problem. In medically advanced countries about $5-10 \%$ of patients with AIDS develope cryptococcosis (Subramanian \& Mathai, 2005); this infection rate is higher in developing countries and reaches 13-45\% (Hakim et al., 2000). Cryptococcocal infection was also documented in $2.8 \%$ of solid transplant recipients with a mortality rate near $42 \%$ (Husain et al., 2001). The most important virulence factors of $C$. neoformans are: capsule production, melanin synthesis and the ability to grow at $37^{\circ} \mathrm{C}$ (Perfect, 2006).

\section{Capsule formation}

A thick polysaccharide capsule can be obtained by $C$. neoformans during lung infection, in contrast to the natural environment where it is weakly encapsulated. After the invasion, C. neoformans can be rehydrated and acquires the capsule composed of glucuronoxylomannan (GXM) (Buchanan $\&$ Murphy, 1998) made of unbranched chains of $\alpha$ 1,3-linked mannose units substituted with $\beta-1,2-$ and $\beta$-1,4-xylose and $\beta$-1,2-glucuronic acid residues and connected with the fungal cell wall through glucan bridges (Bhattacharjee et al., 1984; Todaro-Luck et al., 1989). $\alpha-1,3-$ Glucan found in the cryptococcal cell wall is very important for these interactions (Reese \& Doering, 2003). This structure is similar in composition among different serotypes of $C$. neoformans but differs in antigenicity (McFadden et al., 2007). At least two genes, CAP59 and CAP64, are necessary for capsule formation (Chang \& Kwon-Chung, 1994; Chang et al., 1996). The capsule has many important properties and functions; due to its negative charge it shields the pathogen from phagocytosis and killing by neutrophils, monocytes and macrophages. It is also responsible for complement depletion, antibody unresponsiveness and dysregulation of cytokine secretion by monocytes and macrophages, including TNF $\alpha$, IL-1 $\beta$ and IL- 6 . The capsule can inhibit the migration of leukocytes from the bloodstream to the inflammation sites (Buchanan \& Murphy, 1998; Perfect, 2006). Though the capsule has evolved as a $C$. neoformans defense against adverse environmental conditions such as amoeba attack, it is also useful for invasion of the human organism, facilitating development of infection.

\section{Melanogenesis}

The presence of melanin, a grayish, brown or black pigment, in the C. neoformans cell wall may be a result of fungal adaptation to environmental changes (Plonka \& Grabacka, 2006). It provides protection against ultraviolet radiation, extreme temperatures and other adverse environmental conditions. However, the ability to produce melanin is also one of the important cryptococcal virulence factors, which facilitates fungal escape from lungs and infection of the central nervous system (Perfect, 2006). C. neoformans has a simple pathway for melanogenesis, but it must be able to acquire substrates, diphenolic compounds, from the surroundings because it lacks the tyrosinase enzyme (Fig. 3) (Polacheck \& Kwon-Chung, 1988; Torres-Guererro \& Edman, 1994). The construction and character of the produced pigment depends on the chemical structure of substrates, thus melanins synthesized from $o$-diphenols with hydroxyl groups in the 2,3- or 3,4-positions are usually dark and connected with the cell wall while melanins produced from $p$-diphenols with hydroxyl groups in the 1,4- or 2,5-positions are soluble (Chaskes \& 


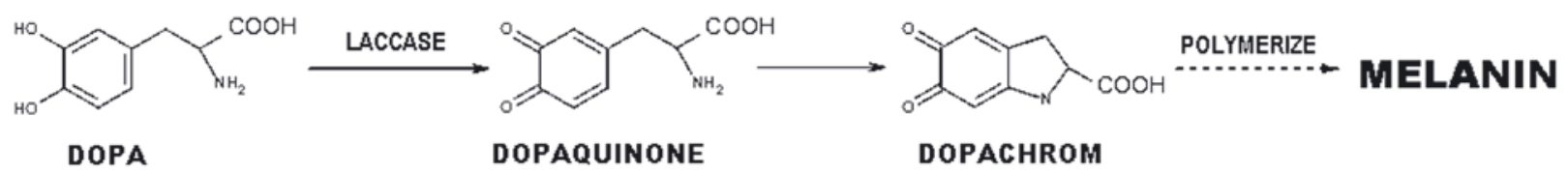

Figure 3. Cryptococcus neoformans melanogenesis pathway. After Buchanan and Murphy (1998), modified.

Tyndall, 1975). A phenoloxidase called laccase is the crucial cooper-containing enzyme responsible for the conversion of diphenolic compounds to dopaquinone, followed by their polymerization to melanin (Williamson, 1994). Laccase is anchored in the fungal cell wall (Polacheck et al., 1982). There are two paralog genes, CNLAC1 and CNLAC2, encoding laccase (Zhu \& Williamson, 2004). During central nervous system infection, $C$. neoformans may use neurotransmitters such as dopamine, norepinephrine and epinephrine as substrates for melanin production (Casadevall et al., 2000). Some properties of melanin facilitate fungal survival during infection. It protects fungi from reactive oxygen species and plays a role as an antioxidant. It is also responsible for cell wall integrity and negative charge, and may be important in protection against antifungal agents and in binding of different ions (including iron) to the cell surface. Melanin helps C. neoformans escape the action of antifungal agents and to abrogate antibody-mediated phagocytosis (Buchanan \& Murphy, 1998; Casadevall et al., 2000; Perfect, 2006).

\section{Mannitol production}

Infection of the central nervous system caused by $C$. neoformans is often associated with production of a large amount of the hexitol D-mannitol by this organism (Liappis et al., 2008). This process may facilitate the development of meningoencephalitis, because mannitol increases the osmolality of the surrounding fluid, thus it may contribute to brain edema, and also prevents oxidative damage to the fungus (Wong et al., 1990). Polymorphonuclear neutrophils (PMN) can kill C. neoformans cells by generating toxic oxygen metabolites such as $\mathrm{OH}^{\cdot}$ and $\mathrm{HOCl}$, which are thought to be key effector molecules against $C$. neoformans, but production of large amounts of mannitol can protect the fungi from oxidative killing by PMN or by cell-free oxidants (Chaturvedi et al., 1996a). Mannitol production is also thought to be helpful for the pathogen to resist other environmental stresses, since Chaturvedi et al. (1996b) showed that a C. neoformans mutant producing low levels of mannitol was more susceptible to heat stress and osmotic stress. The crucial enzyme for mannitol production by this pathogenic species is mannitol dehydrogenase (Perfect et al., 1996).

\section{Adhesion to host cells and crossing the blood-brain barrier}

When addressing the problem of C. neoformans adhesion to the host cells and proteins it is crucial to recognize all factors which have an influence on fungal pathogenicity, but still the mechanism responsible for adhesion and adhesive structures present on the fungal cell wall, remain unknown. It was proven by Chang et al. (2004) that cryptococcal infection of the central nervous system must be preceded by adhesion to the human brain microvascular endothelial cells (HBMEC), followed by transcellular crossing of the blood-brain barrier without disrupting the monolayer integrity. Chang and coworkers showed that there no $C$. neoformans cells were present between HBMEC, so they excluded the possibility of crossing the blood-brain barrier by $C$. neoformans via a paracellular mechanism. However, Olszewski et al. (2004) suggested that urease production by C. neoformans cells facilitates microcapillaries sequestration and disruption of endothelial cells and, in consequence, crossing the blood-brain barrier via a paracellular mechanism. Other hypothetical mechanisms of central nervous system invasion by C. neoformans have been put forward. Santangelo et al. (2004) and Charlier et al. (2009) postulated that C. neoformans cells could invade the central nervous system by means of infected immune cells which could carry the pathogen within through the blood-brain barrier (a "Trojan horse" mechanism). Those authors hypothesized that phagocytosed C. neoformans cells after delivery into the brain tissues can escape from phagocytes and continue invasion and tissue damage due to the acidification of the environment and activation of extracellular phospholipases. C. neofromans has other properties which help in the invasion of the central nervous system. According to Charlier et al. (2005), the size of C. neoformans cells and the modifications of the fungal capsule during central nervous system invasion suggest the existence of an active mechanism that may be triggered during or after crossing of the blood-brain barrier. C. neoformans might induce considerable morphological changes and actin reorganization after adhesion to the HBMEC surface, facilitating engulfment of the fungus by endothelial cells or alteration in tight junctions' permeability (Chen et al., 2003). The mechanism of cryptococcal invasion of the central nervous system 
is still unknown and requires further investigation, and so are the fungal properties that facilitate crossing the blood-brain barrier.

\section{Other virulence factors}

Probably at some stage of infection C. neoformans produces and secretes hydrolytic enzymes, such as proteases and phospholipases, which play a role in nutrition and tissue damage. Cryptococcal extracellular phospholipase exhibits phospholipase B (PLB), lysophospholipase (LPL) and lysophospholipase-transacylase (LPTA) activities (Chen et al., 2000). The action of this enzyme can result in destabilization and destruction of the membranes and lung surfactant, cell lysis and release of lipid second messengers (Ghannoum, 2000; Cox et al., 2001). The phospholipase also enhances the adhesion of $C$. neoformans cells to the lung epithelium (Ganendren et al., 2006). The activity of the phospholipase is strongly correlated with cryptococcal virulence, as proved by Cox et al. (2001) who constructed plb1 mutants which were significantly less virulent in animal models and had a growth defect in a macrophage-like cell line. Probably PLB plays an important role in intracellular growth, survival and replication of $C$. neofromans within macrophages (Santangelo et al., 2004). One factor which might be involved in the survival of $C$. neoformans in the presence of macrophages is the production of eicosanoids: prostaglandins and leukotrienes, which can down-regulate macrophage functions (Noverr et al., 2003). Moreover, the phospholipase plays a role not only in the turnover of cryptococcal cell membrane but also in the maintenance of cell wall integrity and therefore fungal survival, particularly during heat stress (Siafakas et al., 2007). It is also known that $C$. neoformans possesses a proteolytic activity, but these findings must be still investigated (Buchanan \& Murphy, 1998). Recently, a gene for aspartic proteinase was characterized and the three-dimensional structure of this protein was proposed (Pinti et al., 2007). This protein, called CnAP1, has two homologous domains, each containing an Asp residue in a conserved position (Pinti et al., 2007). The fungal resistance to reactive oxygen species is not only due to the mannitol and melanin production, but also very important is the production of $\mathrm{Cu}, \mathrm{Zn}$ superoxide dismutase, peroxidases, glutathione peroxidase and glutathione reductase, which play a role in resistance to oxidative and nitrosative species (Brown et al., 2007b).

C. neoformans can also undergo phenotypic switching, when parent smooth colonies are changed into mucoid colonies which are more virulent during lung infection and can modify the immunological host response (Guerrero \& Fries, 2008). Another very important virulence factor of $C$. neoformans is its ability to grow in the host's physiological conditions, i.e. at $37^{\circ} \mathrm{C}$ in an atmosphere of approx. $5 \%$ $\mathrm{CO}_{2}$ and a $\mathrm{pH}$ higher than 7.0 (Buchanan \& Murphy, 1998). For the fungal survival in the host organism the presence of an intact gene for calcineurin A is essential. Calcineurin A is a phosphoserine-phosphothreonine specific phosphatase encoded by the CNA1 gene (Odom et al., 1997). After activation by pathways connected with fungal stress response calcineurin can dephosphorylate specific proteins responsible for pathogenicity and for growth within the host organism, but specific targets of this enzyme need still to be investigated. Disruption of the CNA1 gene can damage the fungal ability to grow at $37^{\circ} \mathrm{C}$ without affecting the ability to grow at $24^{\circ} \mathrm{C}$ (Odom et al., 1997).

C. neoformans is also able to acquire iron from its environment during tissue and bloodstream infections. The pathway of signaling of iron presence in the surroundings and iron acquisition is only partially understood. Reductases present at the fungal cell surface can reduce ferric to ferrous iron. This process is followed by transport of the ferrous ions into the cell. This process is mediated by a permease (Cft1) and ferroxidase (Cfo1) complex connected with the plasma membrane (Jung \& Kronstad, 2008). The siderophore transporter Sit1, the cell wall protein Cig1 and the mitochondrial proteins Frr1, 3 and 4 also play roles in iron delivery and homeostasis. The Cir1 protein controls the transcription of genes encoding those proteins (Jung \& Kronstad, 2008).

\section{ASPERGILLUS FUMIGATUS}

Aspergillus fumigatus is an ascomycetous, saprophytic and ubiquitous fungus responsible for moulding. It is found worldwide and its small-sized conidia $(2-3 \mu \mathrm{m})$ are abundant in the environment (Gniadek \& Macura, 2007). Airborne A. fumigatus conidia are inhaled by everyone, because their concentration in the air is high, approx. 1-100 conidia per $\mathrm{m}^{3}$ (Latgé, 2001). It is also well known that $A$. fumigatus conidia are frequently present in food, especially in pepper and tea (Bouakline et al., 2000), in tap water (Warris et al., 2003), at home (Ren et al., 2001) and in the office rooms (Buczyńska et al., 2007). This may be the reason why nosocomial acquired infections and community acquired infections quite often develop in immunocompromised as well as in immunocompetent people (Clancy \& Nguyen, 1998). In an immunocompetent person, the efficient immune system is usually able to get rid of these conidia, but sometimes even those people may contract invasive pulmonary aspergillosis (Clancy \& Nguyen, 1998). However, especially patients with severely impaired immunity may contract a life-threatening invasive 
pulmonary disease, disseminated infections or central nervous system infection (Latgé, 2001; Rhodes \& Brakhage, 2006). A high mortality rate, from $60 \%$ to $90 \%$, is correlated with late diagnosis and relatively poor knowledge about fungal pathogenicity and virulence factors (Tekaia \& Latgé, 2005; Rementeria et al., 2005). There are some features thought to be putative virulence factors, including heat tolerance, adhesins, pigment production, toxic metabolites and extracellular enzymes, but most of them evolved as fungal protection against adverse environmental conditions and their role in pathogenicity is often unclear (Alp \& Arikan, 2008). The functions and character of the putative virulence factors of $A$. fumigatus are still under investigation; probably there is a substantial co-operation between these properties, many of which are regulated by numerous genes, so it is often impossible to indicate without doubts which of them contribute to the mechanism of pathogenesis.

\section{Melanin production}

A. fumigatus conidia are often black or grey due to the presence in the cell wall of a pigment called DHN-melanin, which is synthesized from acetate with participation of enzymatic products of six genes (Fig. 4) (Tsai et al., 1999; Latgé, 2001). The melanin functions are protection against ultraviolet radiation, enzymatic lysis, extreme temperatures, as well as against reactive oxygen species during infection (Rementeria et al., 2005).

\section{Secreted hydrolytic enzymes}

A. fumigatus produces and secretes various hydrolytic enzymes, including serine and aspartic protease, metalloproteinase, dipeptydylpeptidases and phospholipases, which contribute to fungal virulence facilitating lung and other tissue colonization. There is a considerable correlation between phos- pholipase activity and severity of infection (Alp \& Arikan, 2008). The serine proteinase and metalloproteinase have an elastinolytic activity, so lungs being rich in elastin, the $A$. fumigatus ability to degrade this protein is important during pulmonary infections (Hogan et al., 1996). The serine proteinase (AFA1p) is a member of subtilisin family and can degrade not only elastin but also collagen, fibrin and fibrinogen. This protein has an extracellular location, but it is also connected with the cell wall (Moutaouakil et al., 1993; Tomee \& Kauffman, 2000). Apart from the mentioned activities, other hydrolytic enzymes are produced by A. fumigatus, including nucleases and phosphatases (Tomee \& Kauffman, 2000).

\section{Toxins}

Secretion of different kinds of toxins to the environment or during infection within the human organism is one of the characteristic features of $A$. fumigatus. The well-known A. fumigatus toxin aflatoxin, which has hepatotoxic and carcinogenic features probably is not produced in the human organism during infection, because its expression is regulated by many genes under complex influence of environmental conditions (OBrian et al., 2003; Rementeria et al., 2005). The most important toxin with a well-proven in vivo activity is gliotoxin. This secondary metabolite from the epipolythiodioxopiperazine family has immunosuppressive properties, because it can inhibit macrophage phagocytosis, T-cell activation and proliferation, and can induce macrophage apoptosis (Hogan et al., 1996). Gliotoxin is also responsible for slowing ciliary beating in the respiratory tract and for epithelial layer damage, so the fungal cells cannot be efficiently removed from the host organism (Tomee \& Kauffman, 2000). Another toxin secreted in vivo is resticotocin, an 18kDa protein (Aspf1) which cleaves a phosphodiester bond in 28S rRNA of eukaryotic ribosomes (Lamy et al., 1991; Hogan et al., 1996). A. fumigatus also pro-

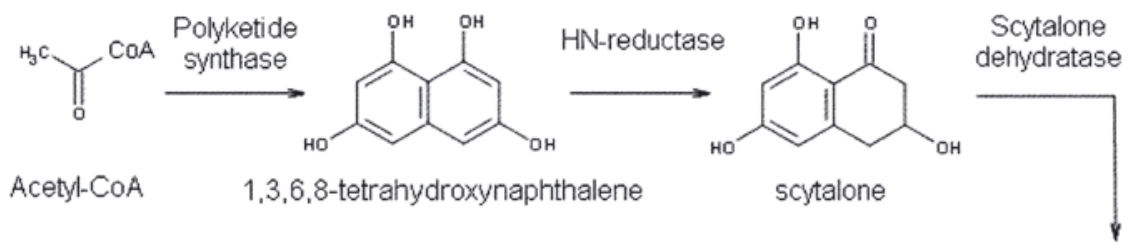

MELANIN<smiles>Oc1cc(O)c2c(O)cccc2c1</smiles>

Figure 4. Pathway for melanin synthesis by Aspergillus fumigatus.

After Latgé (2001), modified. 
duces other immunosuppressive toxins, for example the 14-kDa conidial inhibitory factor and AfD, A. fumigatus diffusible product. Pyrogenic, cytotoxic and shock-evoking activities are also characteristic for other endotoxins produced by $A$. fumigatus, including fumitremorgins, fumagilin, fumagatin and helvolic acid (Tomee \& Kauffman, 2000).

\section{Other virulence factors}

The molecular basis of A. fumigatus pathogenicity is associated with its ability to adhere to host tissues and with binding to different host proteins, including laminin, fibrinogen, surfactant $\mathrm{A}$ and $\mathrm{D}$, complement, immunoglobulin and fibronectin through different receptors connected with the fungal cell wall, which is built of $\alpha, \beta-1,3$-glucan, galactomannan and chitin (Latgé, 2001). The galactomannan can be used as a diagnostic factor, because during infection it can be found in the serum, urine and cerebrospinal fluid (Latgé, 1999; Rementeria et al., 2005). Probably laminin and fibrinogen have a common receptor or distinct receptors, but tightly packed on the surface of conidia (Tomee \& Kauffman, 2000). A. fumigatus conidia are covered with a layer of hydrophobic proteins, called "rodlet layer" built from proteins encoded by at least two genes, $R O D A$ and $R O D B$, responsible for adhesion to albumin and collagen (Latgé, 2001). Protection against reactive oxygen species produced by human immune cells during inflammatory state is also important for developing a fungal infection, therefore A. fumigatus has an ability to produce catalases and superoxide dismutases (Latgé, 2001). Three catalases are expressed by A. fumigatus. CatA, a homodimeric enzyme composed of two $84.5-\mathrm{kDa}$ subunits, is connected with conidia. The other two catalases, Cat1p and Cat $2 p$, are connected with hyphae and have different structures, because the first one is composed of four $90-\mathrm{kDa}$ subunits, and the second is a monomer. Despite the fact that these enzymes can protect $A$. fumigatus against reactive oxygen species in the environment their role as virulence factors during infection is not clear (Paris et al., 2003). Perhaps there are other enzymes with such activity which contribute to the fungal pathogenicity. It has also been proven that A. fumigatus superoxide dismutases, containing $\mathrm{Mn}$ or $\mathrm{Cu}$ and $\mathrm{Zn}$, can efficiently prevent the fungus from oxidative damage (Rementeria et al., 2005). The optimal temperature for $A$. fumigatus growth is $37^{\circ} \mathrm{C}$, but it is able to grow even at $55^{\circ} \mathrm{C}$ and to survive temperatures near $75^{\circ} \mathrm{C}$ (Tekaia \& Latgé, 2005). There are some indications that calcineurin catalytic A subunit, encoded by the CNAA gene, is very important for $A$. fumigatus growth, tissue invasion and pathogenicity (Steinbach et al., 2006).

\section{CONCLUSIONS}

The recent progress in medical techniques, transplantology and antimicrobial treatment is unfortunately the reason of the increasing frequency of invasive fungal infections and high mortality rate, even $90 \%$, among patients with disseminated candidiasis, aspergillosis or cryptococcosis (Richardson, 2005). Additionally, the diagnosis is often difficult because of non-specific symptoms and problems in isolation and identification of fungi (Hinrikson et al., 2005). At present, the majority of pathogenic fungi are susceptible to conventional antifungal treatment, but an increasing resistance to some antifungal drugs is a new, important problem in medicine. Currently used antifungal drugs belong to one of four groups of different character and mechanism of action (Sanglard \& White, 2006). The first group, the polyenes represented by amphotericin B, target ergosterol, a sterol present in the fungal cell membrane, and make pores causing cell death (White et al., 1998). Amphotericin B may be used for treating infections caused by $C$. albicans, C. neoformans and A. fumigatus. The main problem with polyene therapy is that at high and efficient concentrations they are nephrotoxic and must be injected intravenously because of poor solubility (Sanglard \& White, 2006). The second group of antifungal drugs is ergosterol biosynthesis inhibitors, which include azoles, morpholines and allylamines. They can inhibit the late pathway of ergosterol biosynthesis and cell division, causing loss of membrane structure and function (White et al., 1998). Azoles are the most popular drugs from this group, and they can be divided into two classes: imidazoles, which include ketoconazole and clotrimazole used for superficial infections, and triazoles, which include fluconazole, voriconazole and itraconazole used for systemic infections (Sanglard \& White, 2006). Azoles are used for treating candidiasis, but voriconazole is also important for aspergillosis therapy (Macura et al., 2000; Pawlik et al., 2006), whereas fluconazole is used for cryptococcosis therapy because of its ability to cross the blood-brain barrier (Sanglard \& White, 2006). The third group includes inhibitors of nucleic acid synthesis, i.e. 5-flucytosine (Sanglard \& White, 2006). The fourth, the newest category of antifungal drugs, includes echinocandins which target glucan synthase. One echinocandin, caspofungin, is currently used for treatment of Candida and Aspergillus infections; other echinocandins, anidulafungin and micafungin, are in clinical trials (Sanglard \& White, 2006). A serious problem in treatment of fungal infections is the resistance to azoles and 5-flucytosine through a mechanism dependent on alternations in the target enzyme and in drug efflux pumps (Es- 
pinel-Ingroff, 2008). A way to avoid developing resistance is to use multidrug therapy or different antifungal agents, as well as to limit too frequent and uncontrolled usage of the newest category of drugs (Sanglard \& White, 2006). It is also necessary to recognize the mechanism of pathogen-host interactions at the molecular level in order either to prevent the infection or to develop new strategies for therapy and new effective antifungal drugs. Although the knowledge of the fungal pathogenicity and molecular basis of their virulence is already significant, this issue needs further investigation.

\section{REFERENCES}

Albrecht A, Felk A, Pichova I, Naglik JR, Schaller M, de Groot P, Maccallum D, Odds FC, Schäfer W, Klis F, Monod M, Hube B (2006) Glycosylphosphatidylinositol-anchored proteases of Candida albicans target proteins necessary for both cellular processes and hostpathogen interactions. J Biol Chem 281: 688-694.

Al-Fattani MA, Douglas LJ (2006) Biofilm matrix of Candida albicans and Candida tropicalis: chemical composition and role in drug resistance. J Med Microbiol 55: 999-1008.

Alp S, Arikan S (2008) Investigation of extracellular elastase, acid proteinase and phospholipase activities as putative virulence factors in clinical isolates of Aspergillus species. J Basic Microbiol 48: 331-337.

Bader T, Bodendorfer B, Schröppel K, Morschhäuser J (2003) Calcineurin is essential for virulence in Candida albicans. Infect Immun 71: 5344-5354.

Bhattacharjee AK, Bennett JE, Glaudemans CP (1984) Capsular polysaccharides of Cryptococcus neoformans. Rev Infect Dis 6: 619-624.

Blankenship JR, Wormley FL, Boyce MK, Schell WA, Filler SG, Perfect JR, Heitman J (2003) Calcineurin is essential for Candida albicans survival in serum and virulence. Eukaryot Cell 2: 422-430.

Bouakline A, Lacroix C, Roux N, Gangneux JP, Derouin F (2000) Fungal contamination of food in hematology units. J Clin Microbiol 38: 4272-4273.

Brown AJ, Gow NA (1999) Regulatory networks controlling Candida albicans morphogenesis. Trends Microbiol 7: 333-338.

Brown AJ, Odds FC, Gow NA (2007a) Infection-related gene expression in Candida albicans. Curr Opin Microbiol 10: 307-313.

Brown SM, Campbell LT, Lodge JK (2007b) Cryptococcus neoformans, a fungus under stress. Curr Opin Microbiol 10: 320-325.

Buchanan KL, Murphy JW (1998) What makes Cryptococcus neoformans a pathogen? Emerg Infect Dis 4: 71-83.

Buczyńska A, Cyprowski M, Piotrowska M, SzadkowskaStańczyk J (2007) Indoor moulds: results of the environmental study in office rooms. Med $\operatorname{Pr}$ 58: 521-525 (in Polish).

Calderone RA, Fonzi WA (2001) Virulence factors of Candida albicans. Trends Microbiol 9: 327-335.

Casadevall A, Rosas AL, Nosanchuk JD (2000) Melanin and virulence in Cryptococcus neoformans. Curr Opin Microbiol 3: 354-358.

Casanova M, Lopez-Ribot JL, Monteagudo C, LlombartBosch A, Sentandreu R, Martinez JP (1992) Identification of a 58-kilodalton cell surface fibrinogen-binding mannoprotein from Candida albicans. Infect Immun 60: 4221-4229.

Chaffin WL, López-Ribot JL, Casanova M, Gozalbo D, Martínez JP (1998) Cell wall and secreted proteins of Candida albicans: identification, function, and expression. Microbiol Mol Biol Rev 62: 130-180.

Chandra J, Kuhn DM, Mukherjee PK, Hoyer LL, McCormick T, Ghannoum MA (2001) Biofilm formation by the fungal pathogen Candida albicans: development, architecture, and drug resistance. J Bacteriol 183: 53855394.

Chang YC, Penoyer LA, Kwon-Chung KJ (1996) The second capsule gene of Cryptococcus neoformans, CAP64, is essential for virulence. Inf Immun 64: 1977-1983.

Chang YC, Kwon-Chung KJ (1994) Complementation of a capsule-deficient mutation of Cryptococcus neoformans restores its virulence. Mol Cell Biol 14: 4912-4919.

Chang YC, Stins MF, McCaffery MJ, Miller GF, Pare DR, Dam T, Paul-Satyaseela M, Kim KS, Kwon-Chung KJ (2004) Cryptococcal yeast cells invade the central nervous system via transcellular penetration of the bloodbrain barrier. Infect Immun 72: 4985-4995.

Charlier C, Chrétien F, Baudrimont M, Mordelet E, Lortholary O, Dromer F (2005) Capsule structure changes associated with Cryptococcus neoformans crossing of the blood-brain barrier. Am J Pathol 166: 412-432.

Charlier C, Nielsen K, Daou S, Brigitte M, Chretien F, Dromer F (2009) Evidence of a role for monocytes in dissemination and brain invasion by Cryptococcus neoformans. Infect Immun 77: 120-127.

Chaskes S, Tyndall RL (1975) Pigment production by Cryptococcus neoformans from para- and ortho-diphenols: effect of the nitrogen source. J Clin Microbiol 1: 509-514.

Chaturvedi V, Wong B, Newman SL (1996a) Oxidative killing of Cryptococcus neoformans by human neutrophils. Evidence that fungal mannitol protects by scavenging reactive oxygen intermediates. I Immunol 156: 38363840.

Chaturvedi V, Flynn T, Niehaus WG, Wong B (1996b) Stress tolerance and pathogenic potential of a mannitol mutant of Cryptococcus neoformans. Microbiology 142: 937-943.

Chen SC, Wright LC, Golding JC, Sorrell TC (2000) Purification and characterization of secretory phospholipase B, lysophospholipase and lysophospholipase/transacylase from a virulent strain of the pathogenic fungus Cryptococcus neoformans. Biochem J 347: 431-439.

Chen SH, Stins MF, Huang SH, Chen YH, Kwon-Chung KJ, Chang Y, Kim KS, Suzuki K, Jong AY (2003) Cryptococcus neoformans induces alterations in the cytoskeleton of human brain microvascular endothelial cells. $J$ Med Microbiol 52: 961-970.

Clancy CJ, Nguyen MH (1998) Acute community-acquired pneumonia due to Aspergillus in presumably immunocompetent hosts: clues for recognition of a rare but fatal disease. Chest 114: 629-634.

Cox GM, McDade HC, Chen SC, Tucker SC, Gottfredsson M, Wright LC, Sorrell TC, Leidich SD, Casadevall A, Ghannoum MA, Perfect JR (2001) Extracellular phospholipase activity is a virulence factor for Cryptococcus neoformans. Mol Microbiol 39: 166-175.

Crowe JD, Sievwright IK, Auld GC, Moore NR, Gow NA, Booth NA (2003) Candida albicans binds human plasminogen: identification of eight plasminogen-binding proteins. Mol Microbiol 47: 1637-1651.

dos Santos AL, de Carvalho IM, da Silva BA, Portela MB, Alviano CS, de Araújo Soares RM (2006) Secretion of serine peptidase by a clinical strain of Candida albicans: influence of growth conditions and cleavage of human 
serum proteins and extracellular matrix components. FEMS Immunol Med Microbiol 46: 209-220.

Eckert SE, Sheth CC, Mühlschlegel FA (2007) Regulation of morphogenesis in Candida species. In Candida. Comparative and functional genomics. d'Enfert Ch, Hube B, eds, pp 263-291. Caister Academic Press, Norfolk.

d'Enfert C, Hube B (2007) Introduction. In Candida. Comparative and functional genomics. d'Enfert Ch, Hube B, eds, pp 1-5. Caister Academic Press, Norfolk.

Espinel-Ingroff A (2008) Mechanisms of resistance to antifungal agents: yeasts and filamentous fungi. Rev Iberoam Micol 25: 101-106.

Filler SG (2006) Candida-host cell receptor-ligand interactions. Curr Opin Microbiol 9: 333-339.

Filler SG, Sheppard DC, Edwards JE Jr (2006) Molecular basis of fungal adherence to endothelial and epithelial cells. In Molecular principles of fungal pathogenesis. Heitman J, Filler SG, Edwards JE Jr, Mitchell AP, eds, pp 187-196. ASM Press, Washington DC.

Ganendren R, Carter E, Sorrell T, Widmer F, Wright L (2006) Phospholipase B activity enhances adhesion of Cryptococcus neoformans to a human lung epithelial cell line. Microbes Infect 8: 1006-1015.

Ghannoum MA (2000) Potential role of phospholipases in virulence and fungal pathogenesis. Clin Microbiol Rev 13: $122-143$.

Gniadek A, Macura AB (2007) Intensive care unit environment contamination with fungi. Adv Med Sci 52: 283287.

Guerrero A, Fries BC (2008) Phenotypic switching in Cryptococcus neofromans contributes to virulence by changing the immunological host response. Inf Immun 76: 4322-4331.

Hakim JG, Gangaidzo IT, Heyderman RS, Mielke J, Mushangi E, Taziwa A, Robertson VJ, Musvaire P, Mason PR (2000) Impact of HIV infection on meningitis in Harare, Zimbabwe: a prospective study of 406 predominantly adult patients. AIDS 14: 1401-1407.

Hinrikson HP, Hurst SF, De Aguirre L, Morrison CJ (2005) Molecular methods for the identification of Aspergillus species. Med Mycol 43: S129-S137.

Hogan LH, Klein BS, Levitz SM (1996) Virulence factors of medically important fungi. Clin Microbiol Rev 9: 469488.

Hube B, Naglik J (2001) Candida albicans proteinases: resolving the mystery of a gene family. Microbiology 147: 1997-2005.

Husain S, Wagener MM, Singh N (2001) Cryptococcus neoformans infection in organ transplant recipients: variables influencing clinical characteristics and outcome. Emerg Infect Dis 7: 375-381.

Ibrahim AS, Mirbod F, Filler SG, Banno Y, Cole GT, Kitajima Y, Edwards JE Jr, Nozawa Y, Ghannoum MA (1995) Evidence implicating phospholipase as a virulence factor of Candida albicans. Infect Immun 63: 1993-1998.

Jong AY, Stins MF, Huang SH, Chen SH, Kim KS (2001) Traversal of Candida albicans across human blood-brain barrier in vitro. Infect Immun 69: 4536-4544.

Jung WH, Kronstad JW (2008) Iron and fungal pathogenesis: a case study with Cryptococcus neoformans. Cell Microbiol 10: 277-284.

Lamy B, Moutaouakil M, Latgé JP, Davies J (1991) Secretion of a potential virulence factor, a fungal ribonucleotoxin, during human aspergillosis infections. Mol Microbiol 5: 1811-1815.

Latgé JP (1999) Aspergillus fumigatus and aspergillosis. Clin Microbiol Rev 12: 310-350.

Latgé JP (2001) The pathobiology of Aspergillus fumigatus. Trends Microbiol 9: 382-389.
Lengeler KB, Davidson RC, D'souza C, Harashima T, Shen WC, Wang P, Pan X, Waugh M, Heitman J (2000) Signal transduction cascades regulating fungal development and virulence. Microbiol Mol Biol Rev 64: 746-785.

Liappis AP, Kan VL, Richman NC, Yoon B, Wong B, Simon GL (2008) Mannitol and inflammatory markers in the cerebral spinal fluid of HIV-infected patients with cryptococcal meningitis. Eur J Clin Microbiol Infect Dis 27: 477-479.

Lin X, Heitman J (2005) Chlamydospore formation during hyphal growth in Cryptococcus neoformans. Eukaryot Cell 4: 1746-1754.

Lo HJ, Köhler JR, DiDomenico B, Loebenberg D, Cacciapuoti A, Fink GR (1997) Nonfilamentous C. albicans mutants are avirulent. Cell 90: 939-949.

Macura AB, Pawlik B, Szczepko I (2000) The susceptibility of Aspergillus and Penicillium to recent antimycotics. Wiad Parazytol 46: 157-162 (in Polish).

Mavor AL, Thewes S, Hube B (2005) Systemic fungal infections caused by Candida species: epidemiology, infection process and virulence attributes. Curr Drug Targets 6: 863-874.

McFadden DC, Fries BC, Wang F, Casadevall A (2007) Capsule structural heterogeneity and antigenic variation in Cryptococcus neoformans. Eukaryot Cell 6: 14641473.

Mendes A, Mores AU, Carvalho AP, Rosa RT, Samaranayake LP, Rosa EA (2007) Candida albicans biofilms produce more secreted aspartyl protease than the planktonic cells. Biol Pharm Bull 30: 1813-1815.

Mitchell TG, Perfect JR (1995) Cryptococcosis in the era of AIDS - 100 years after the discovery of Cryptococcus neoformans. Clin Microbiol Rev 8: 515-548.

Moutaouakil M, Monod M, Prévost MC, Bouchara JP, Paris S, Latgé JP (1993) Identification of the 33-kDa alkaline protease of Aspergillus fumigatus in vitro and in vivo. J Med Microbiol 39: 393-399.

Naglik JR, Challacombe SJ, Hube B (2003) Candida albicans secreted aspartyl proteinases in virulence and pathogenesis. Microbiol Mol Biol Rev 67: 400-428.

Naglik JR, Albrecht A, Bader O, Hube B (2004) Candida albicans proteinases and host/pathogen interactions. Cell Microbiol 6: 915-926.

Newport G, Agabian N (1997) KEX2 influences Candida albicans proteinase secretion and hyphal formation. J Biol Chem 272: 28954-28961.

Noverr MC, Cox GM, Perfect JR, Huffnagle GB (2003) Role of PLB1 in pulmonary inflammation and cryptococcal eicosanoid production. Infect Immun 71: 1538-1547.

OBrian GR, Fakhoury AM, Payne GA (2003) Identification of genes differentially expressed during aflatoxin biosynthesis in Aspergillus flavus and Aspergillus parasiticus. Fungal Genet Biol 39: 118-127.

Odds FC, Gow NAR, Brown AJP (2006) Toward a molecular understanding of Candida albicans virulence. In Molecular principles of fungal pathogenesis. Heitman J, Filler SG, Edwards JE Jr, Mitchell AP, eds, pp 305-319. ASM Press, Washington DC.

Odom A, Muir S, Lim E, Toffaletti DL, Perfect J, Heitman J (1997) Calcineurin is required for virulence of Cryptococcus neoformans. EMBO J 16: 2576-2589.

Olszewski MA, Noverr MC, Chen GH, Toews GB, Cox GM, Perfect JR, Huffnagle GB (2004) Urease expression by Cryptococcus neoformans promotes microvascular sequestration, thereby enhancing central nervous system invasion. Am J Pathol 164: 1761-1771.

Paris S, Wysong D, Debeaupuis JP, Shibuya K, Philippe B, Diamond RD, Latgé JP (2003) Catalases of Aspergillus fumigatus. Infect Immun 71: 3551-3562. 
Pawlik B, Szul A, Macura AB (2006) Susceptibility of fungi isolated from clinical materials to voriconazole. Med Dosw Mikrobiol 58: 155-161 (in Polish).

Perfect JR, Rude TH, Wong B, Flynn T, Chaturvedi V, Niehaus W (1996) Identification of a Cryptococcus neoformans gene that directs expression of the cryptic Saccharomyces cerevisiae mannitol dehydrogenase gene. J Bacteriol 178: 5257-5262.

Perfect JR, Casadevall A (2002) Cryptococcosis. Infect Dis Clin North Am 16: 837-874.

Perfect JR, Casadevall A (2006) Fungal molecular pathogenesis: what can it do and why do we need it? In Molecular principles of fungal pathogenesis. Heitman J, Filler SG, Edwards JE Jr, Mitchell AP, eds, pp 3-11. ASM Press, Washington DC.

Perfect JR (2006) Cryptococcus neofromans: a sugar-coated killer. In Molecular principles of fungal pathogenesis. Heitman J, Filler SG, Edwards JE Jr, Mitchell AP, eds, pp 281-303. ASM Press, Washington DC.

Phan QT, Fratti RA, Prasadarao NV, Edwards JE Jr, Filler SG (2005) N-cadherin mediates endocytosis of Candida albicans by endothelial cells. J Biol Chem 280: 1045510461.

Pinti M, Orsi CF, Gibellini L, Esposito R, Cossarizza A, Blasi E, Peppoloni S, Mussini C (2007) Identification and characterization of an aspartyl protease from Cryptococcus neoformans. FEBS Lett 581: 3882-3886.

Plonka PL, Grabacka M (2006) Melanin synthesis in microorganisms-biotechnological and medical aspects. Acta Biochim Polon 53: 429-443.

Polacheck I, Hearing VJ, Kwon-Chung KJ (1982) Biochemical studies of phenoloxidase and utilization of catecholamines in Cryptococcus neoformans. J Bacteriol 150: 1212-1220.

Polacheck I, Kwon-Chung KJ (1988) Melanogenesis in Cryptococcus neoformans. J Gen Microbiol 134: 1037-1041.

Ramage G, Ghannoum MA, Lopez-Ribot JL (2006) Fungal biofilms: agents of disease and drug resistance. In Molecular principles of fungal pathogenesis. Heitman J, Filler SG, Edwards JE Jr, Mitchell AP, eds, pp 177-185. ASM Press, Washington D.C

Rapala-Kozik M, Karkowska J, Jacher A, Golda A, Barbasz A, Guevara-Lora I, Kozik A (2008) Kininogen adsorption to the cell surface of Candida spp. Int Immunopharmacol 8: 211-216.

Reese AJ, Doering TL (2003) Cell wall $\alpha-1,3-$ glucan is required to anchor the Cryptococcus neoformans capsule. Mol Microbiol 50: 1401-1409.

Rementeria A, López-Molina N, Ludwig A, Vivanco AB, Bikandi J, Pontón J, Garaizar J (2005) Genes and molecules involved in Aspergillus fumigatus virulence. Rev Iberoam Micol 22: 1-23.

Ren $\mathrm{P}$, Jankun TM, Belanger $\mathrm{K}$, Bracken MB, Leaderer BP (2001) The relation between fungal propagules in indoor air and home characteristics. Allergy 56: 419-424.

Rhodes JC, Brakhage AA (2006) Molecular determinants of virulence in Aspergillus fumigatus. In Molecular principles of fungal pathogenesis. Heitman J, Filler SG, Edwards JE Jr, Mitchell AP, eds, pp 333-345. ASM Press, Washington DC.

Richardson MD (2005) Changing patterns and trends in systemic fungal infections. J Antimicrob Chemother 56: 5-11.

Ruiz-Herrera J, Elorza MV, Valentín E, Sentandreu R (2006) Molecular organization of the cell wall of Candida albicans and its relation to pathogenicity. FEMS Yeast Res 6: 14-29.

Sanglard D, White TC (2006) Molecular principles of antifungal drug resistance. In Molecular principles of fun- gal pathogenesis. Heitman J, Filler SG, Edwards JE Jr, Mitchell AP, eds, pp 197-212. ASM Press, Washington D.C

Santangelo R, Zoellner H, Sorrell T, Wilson C, Donald C, Djordjevic J, Shounan Y, Wright L (2004) Role of extracellular phospholipases and mononuclear phagocytes in dissemination of cryptococcosis in a murine model. Infect Immun 72: 2229-2239.

Schaller M, Borelli C, Korting HC, Hube B (2005) Hydrolytic enzymes as virulence factors of Candida albicans. Mycoses 48: 365-377.

Sheppard DC, Yeaman MR, Welch WH, Phan QT, Fu Y, Ibrahim AS, Filler SG, Zhang M, Waring AJ, Edwards JE Jr (2004) Functional and structural diversity in the Als protein family of Candida albicans. J Biol Chem 279: 30480-30489.

Sia RA, Lengeler KB, Heitman J (2000) Diploid strains of the pathogenic basidiomycete Cryptococcus neoformans are thermally dimorphic. Fungal Genet Biol 29: 153-163.

Siafakas AR, Sorrell TC, Wright LC, Wilson C, Larsen M, Boadle R, Williamson PR, Djordjevic JT (2007) Cell wall-linked cryptococcal phospholipase B1 is a source of secreted enzyme and a determinant of cell wall integrity. J Biol Chem 282: 37508-37514.

Singleton DR, Masuoka J, Hazen KC (2001) Cloning and analysis of a Candida albicans gene that affects cell surface hydrophobicity. J Bacteriol 183: 3582-3588.

Slutsky B, Buffo J, Soll DR (1985) High-frequency switching of colony morphology in Candida albicans. Science 230: 666-669.

Slutsky B, Staebell M, Anderson J, Risen L, Pfaller M, Soll DR (1987) "White-opaque transition": a second highfrequency switching system in Candida albicans. J Bacteriol 169: 189-197.

Staab JF, Bradway SD, Fidel PL, Sundstrom P (1999) Adhesive and mammalian transglutaminase substrate properties of Candida albicans Hwp1. Science 283: 1535-1538.

Steinbach WJ, Cramer RA Jr, Perfect BZ, Asfaw YG, Sauer TC, Najvar LK, Kirkpatrick WR, Patterson TF, Benjamin DK Jr, Heitman J, Perfect JR (2006) Calcineurin controls growth, morphology, and pathogenicity in Aspergillus fumigatus. Eukaryot Cell 5: 1091-1103.

Subramanian S, Mathai D (2005) Clinical manifestations and management of cryptococcal infection. J Postgrad Med 51: S21-S26.

Tekaia F, Latgé JP (2005) Aspergillus fumigatus: saprophyte or pathogen? Curr Opin Microbiol 8: 385-392.

Theiss S, Ishdorj G, Brenot A, Kretschmar M, Lan CY, Nichterlein T, Hacker J, Nigam S, Agabian N, Köhler GA (2006) Inactivation of the phospholipase B gene PLB5 in wild-type Candida albicans reduces cell-associated phospholipase A2 activity and attenuates virulence. Int J Med Microbiol 296: 405-420.

Todaro-Luck F, Reiss E, Cherniak R, Kaufman L (1989) Characterization of Cryptococcus neoformans capsular glucuronoxylomannan polysaccharide with monoclonal antibodies. Infect Immun 57: 3882-3887.

Tomee JFC, Kauffman HF (2000) Putative virulence factors of Aspergillus fumigatus. Clin Exp Allergy 30: 476-484.

Torres-Guererro H, Edman JC (1994) Melanin-deficient mutants of Cryptococcus neoformans. J Med Vet Mycol 32: 303-313.

Tsai HF, Wheeler MH, Chang YC, Kwon-Chung KJ (1999) A developmentally regulated gene cluster involved in conidial pigment biosynthesis in Aspergillus fumigatus. J Bacteriol 181: 6469-6477.

Warris A, Klaassen CH, Meis JF, De Ruiter MT, De Valk HA, Abrahamsen TG, Gaustad P, Verweij PE (2003) Molecular epidemiology of Aspergillus fumigatus iso- 
lates recovered from water, air, and patients shows two clusters of genetically distinct strains. J Clin Microbiol 41: 4101-4106.

Weissman Z, Kornitzer D (2004) A family of Candida cell surface haem-binding proteins involved in haemin and haemoglobin-iron utilization. Mol Microbiol 53: 12091220.

White TC, Agabian N (1995) Candida albicans secreted aspartyl proteinases: isoenzyme pattern is determined by cell type, and levels are determined by environmental factors. J Bacteriol 177: 5215-5221.

White TC, Marr KA, Bowden RA (1998) Clinical, cellular, and molecular factors that contribute to antifungal drug resistance. Clin Microbiol Rev 11: 382-402.

Whiteway M, Bachewich C (2006) Signal transduction in the interactions of fungal pathogens and mammalian hosts. In Molecular principles of fungal pathogenesis. Heitman J, Filler SG, Edwards JE Jr, Mitchell AP, eds, pp 143-161. ASM Press, Washington DC.
Wickes BL, Mayorga ME, Edman U, Edman JC (1996) Dimorphism and haploid fruiting in Cryptococcus neoformans: association with the alpha-mating type. Proc Natl Acad Sci USA 93: 7327-7331.

Williamson PR (1994) Biochemical and molecular characterization of the diphenol oxidase of Cryptococcus neoformans: identification as a laccase. J Bacteriol 176: 656-664.

Wong B, Perfect JR, Beggs S, Wright KA (1990) Production of the hexitol D-mannitol by Cryptococcus neoformans in vitro and in rabbits with experimental meningitis. Infect Immun 58: 1664-1670.

Yang YL (2003) Virulence factors of Candida species. J Microbiol Immunol Infect 36: 223-228.

Zhu X, Williamson PR (2004) Role of laccase in the biology and virulence of Cryptococcus neoformans. FEMS Yeast Res 5: 1-10. 